\title{
AN INTERFEROMETRIC OBSERVATION OF THE NGC 7538 MOLECULAR CLOUD CORE
}

Osamu KAMEYA (National Astronomical Observatory, Mizusawa), Ryohei KAWABE, koh-Ichiro MORITA, Masato ISHIGURO (National Astronomical observatory, Nobeyamal, and NaOmi HIRANO ( $H$ i cotsubashi University)

A high-spatial-resolution observation of the NGC 7538 molecular cloud core has been performed with the Nobeyama Millimeter Array. We report on the detailed structure of the region including IRS1-3 complex and IRS11 based on the CS $J=1-0$ line observational results.

The observation was done in December. 1988. The field center was at R.A. (1950)=23h11m36.8s, Dec $(1950)=61^{\circ} 11^{\prime} 10^{\prime \prime}$ which is between IRS1-3 complex and IRS11. The primary beam, 2.5' (FWHM), was large enough to cover both IRS1-3 complex and IRST1. We used 18 baselines, and the synthesized beam became 10.6" $\times 10.4^{\prime \prime}$ (natural weight).

We have found five high-density regions $\left(>10^{4} \mathrm{~cm}^{-3}\right)$. One of them has a maximum at the 420 maser/continuum source near IRS11. This result suggests that star formation activity ocours at the position of the H20 maser/continuum source (Kameya et al. 1990). The co bipolar outflow near IRS11 (Kameya et al. 1989) originates the CS peak position. On the other hands, IRS11 itself is not at the peak and it seems to stay in the blue lobel of the co outflow. Therefore IRS11 itself is probably an infrared reflection nebula which is in a cavity in a blue lobe of the bipolar molecular outflow originating at the 120 maser/continuum source.

On the other hand, the cS emission dose not have a maximum at IRS1-3 complex. The cS $J=1-0$ structure at IRS1-3 complex is different from that of CS $J=2-1$ (Kawabe et al. 1992): there is no shell like structure. This may because the optical depth of CS J=1-0 is too large to make a contract of CS $J=1-0$ intensity, and the central high-density region $\left(n(\mathrm{H} 2)>10^{5} \mathrm{~cm}^{-3}\right.$ ) is probably hidden in the medium density region ( $n(H 2)$ is about $10^{4} \mathrm{~cm}^{-3}$ ).

References

Kameya, O., Hasegawa, T.I., Hirano, N., Takakubo, K., and Seki, M., 1989, Ap.J., 339, 222.

Kameya, 0.. Morita, K.I., Kawabe, R., and Ishiguro, M., $1990, A p . J ., 355,562$.

Kawabe, R., Suzuki, M., Hirano, N., Akabane, K. Barsony, M. Najita, J.R., Kameya, 0., and Ishiguro, M., 1992, PASJ, 44, 435 . 